\title{
Introduction of the distributed registry technology into the company's management system
}

\author{
Nikolay Mrochkovsky, ${ }^{1, *}$ \\ ${ }^{1}$ Plekhanov Russian University of Economics, 117997, Stremyanny lane, 36, Moscow, Russia
}

\begin{abstract}
The technology of the distributed registry creates new opportunities for changing the management system, which are discussed in the article. The use of the blockchain allows building a decentralized autonomous commercial organization on a network basis. With the help of $\mathrm{p} 2 \mathrm{p}$ technology, it is possible to radically reorganize the structure of organization and management, and reduce the management and transaction costs inherent in the traditional hierarchical structure of the firm. The analysis of the steps by means of which real enterprises can be transferred to the blockchain is provided, and the role of smart contracts is analyzed.
\end{abstract}

Currently, the blockchain, that is, the technology of the distributed registry, is the most important and new breakthrough technology. It allows using data for new purposes, for implementing new ideas that is the essence of the digital economy, as indicated in the Digital Economy Development Program [1]. The new use of data consists in applying them not only for educational and scientific purposes, but also in entrepreneurship. The development of the Internet, computers and mobile devices and the formation of $p 2 p$ technology on this basis provides new and huge opportunities for the development of entrepreneurship.

The purpose of the article is to determine what changes in entrepreneurship are formed through the blockchain technology, what the decentralized autonomous organizations in business are, and how they are created.

The technology of blockchain is the result of the crypto-technical revolution and the development of the Internet. The blockchain is a peer-to-peer network that has an open source protocol that does not require centralized servers to verify transactions and data in the network. Its essential feature is that this technology allows transmitting not only the information but also the value in the form of machine money - cryptocurrencies, which are created during the confirmation of transactions by cryptographic encryption of the codes of transaction units. These two sides - the ability to make and confirm direct transactions between economic agents without intermediaries and transfer of value - give grounds to call this technology subversive. Because of its subversive nature, it has a revolutionary effect on various aspects of society, including entrepreneurship and business.

The blockchain allows organizing interaction of economic agents on the basis of intellectual technology, and not on the basis of legal contracts, allows ensuring cooperation

${ }^{*}$ Corresponding author: nikolay@ mrochkovskiy.ru 
not on the basis of personal acquaintance and social trust, but on the implementation of the technological basis of joint activities. The blockchain creates an opportunity to reach a consensus based on technology, rather than contracts. Hence, trust between the participants is formed not on the social, and, therefore, evaluation, basis, but on the basis of machine technology. Therefore, the blockchain is called the "machine of trust" [2,3]. In this case, we have a new stage in the development of intellectual entrepreneurship. R. Chervitz defined "intellectual entrepreneurship" as the production of new knowledge and their embodiment in concrete life benefits through mechanisms of cooperation and interaction of economic agents, which in practice take the form of various intellectual communities [4]. In this case, the development of connections is carried out using the capabilities of artificial intelligence to automate those processes that can be given to the machine technology.

Decentralization of business is one of the manifestations of decentralization introduced by the blockchain technology. Decentralization of business occurs through the emergence of the distributed autonomous organizations (Distributed Autonomous Organizations, DAO), which are managed and operate on the blockchain. A decentralized organization is also called an autonomous corporation (DAC). Cryptocurrencies, deregulation of crowd-hosting and the separation of services lie at the heart of the emergence of DAO.

The essence of DAO is that management of the organization is not entrusted to a centralized management body but to a set of business rules developed and formed in a specific service that allows the organization "working without the participation of a person solely under the control of an incorruptible set of business rules" controlled by automation [5]. This is not anarchy, but management based on the developed software, and the possibility of using (later) artificial intelligence. Most of the main blockchain platforms were developed using a transparent, open source, a collective approach and a large amount of decentralized work.

Let us consider the content of the concept of DAO. The concept of DAO is borrowed from the field of artificial intelligence. In this concept, the network of autonomous agents performs tasks that can be created in a model of a corporation that operates without the participation of man under control of a set of business rules.

DAO is a new paradigm of economic cooperation [6]. Such an organization is fundamentally different from the usual company in its decentralized structure and autonomy. Decentralization means that the company is built horizontally. It does not have a sole proprietor or a board of directors. Each member of the organization is a full-fledged co-owner and has equal powers and unlimited access to information.

A similar structure is provided by the blockchain. The blockchain in this case is an electronic register of the company, which is maintained and certified by all network members. Autonomy is in the fact that the organization does not depend on traditional financial and political institutions. The use of cryptocurrency instead of conventional money plays an important role in this. Cryptocurrency in the blockchain acts in different hypostases - as a protocol, as software, and as a blockchain system. In this sense, it itself can be classified as DAO, since it has a decentralized structure, and most processes in it are autonomous [7].

Examples of DAO. The decentralized cloud storage network Storj can be referred as an example of a long-working and successfully operating DAO. Mistrust of users to centralized clouds is primarily due to the fact that in recent years, there have been more cases of information leakage from cloud servers. Security researchers are almost daily signaling about unprotected cloud servers. In July 2017, a leak of personal data of about 14 million customers from the cloud repository operator Verizon took place. In Russia, in 2017, the biggest leak happened - a database with information on more than 5.6 million customers of insurance companies [7]. (The main leakage of 2017. https://www.infowatch.ru/analytics/digest/19546). The number of leaks of confidential information in Russia in 2016 was more than the level of 2015 by $80 \%$, and only in 2016 
there were 213 such cases. On these indicators, Russia is ranked the second one after the United States (838 cases). The expert E. Nabigaev believes: "Such a significant percentage is associated with a low level of awareness in the matters of information security among companies' personnel. Employees do not often know that is impermissible to use cloud services for the exchange of confidential information in the corporate sphere" [8].

Storj's decentralized cloud storage network with applications allows users renting out unused hard disk space and storing files on the network. Storj splits the files into parts, encrypts them and distributes along the peer-to-peer network, which provides a much greater level of security. The project has its own SJCX token, the cryptocurrency, which is used to purchase space on the network and pay for the services of the provider. In addition, superfluous SJCX can be exchanged on cryptocurrency exchanges [6].

Another example - Steemit, is a social media platform, similar to Reddit. But, unlike Reddit, the project is based on the technology of blockchain and has its own cryptocurrency. Users earn Steem-dollars by publishing articles and images, and the amount of compensation is determined by the reaction of the community (likes, recommendations, etc.) on this or that material. This is a censorship-free platform, where the only moderator is the community of the project. In 2016, the Russian-speaking fork of the platform that is called Golos (Voice) also appeared [6].

New ideas for DAO were proposed as a result of the competition for the best idea of DAO, which the ForkLog platform hosted in 2016. 30 bids of various properties were submitted for the competition. As winners, the following projects were selected: the inheritance of digital data; the creation of charitable companies based on the blockchain; exchange of the computing facilities; automation of settlements in stores; platform for the interaction of consumers and producers of agricultural products; ICO market regulation project; the project of distribution of natural riches of Russia between citizens; business card holder on the blockchain; investment portfolio; and the project of the state of noonauts on the blockchain [9]. Thus, the idea of DAO can be realized in various directions.

The implementation of the blockchain and the transition to the principles of DAO can be carried out:

a) within existing organizations as an additional technology;

b) outside the organization, as a startup, which is not concerned by the existing processes.

Thus, it is possible to create DAO from scratch as a startup, and also the functions and work of real enterprises can be rethought.

There are many functions related to local legislation in the work of enterprises, such as registration, insurance, taxation, and licensing. When moving to the blockchain, autonomous enterprises can be created in the cloud, operating on the basis of the blockchain and smart contracts that could independently conclude contracts, register under any jurisdiction, under which they can work.

There are two main ways of creating DAO:

1. Introduction of principles or partial elements of the DAO design into a traditional organization.

2. The combination of autonomous agents on the basis of special programs using artificial intelligence.

Let us outline the stages of the transition from autonomous agents to DAO:

Stage 1 . Users voluntarily and independently participate in solving unrelated tasks.

Stage 2. Creation of teamwork. Users interact on the basis of giving value to the achievement of common goals or objectives.

Stage 3. Users are cooperating, assuming they receive part of the total profit.

Stage 4. Spreading functions to a wider network by advertising these functions.

Stage 5. Decentralization. There is further scalability by creating additional capacities for the periphery. 
Stage 6. Autonomy. Autonomous agents work on the basis of smart contracts and (later) artificial intelligence. Smart contract algorithms provide self-sufficiency in operations and creation of values in the centers, on the periphery and in the arteries of the organization.

Let us consider the measures that should be taken to implement the project and introduce DAO:

1. Development of the architecture of the DAO, which holds distributed and decentralized actions.

2. Determination of the options of participation in the DAO.

In turn, options for participation in the DAO are different: buying shares, buying cryptocurrency, and buying or earning tokens.

Options for earning tokens can also be different: active or passive work.

Active work that is the solution of any task claimed by the DAO for a particular project, such as error search, software development, voting, etc.

Passive work that is usually done by sharing something, such as computer processing cycles, Internet access, storage or even sharing one's data.

3. Obtaining units of value.

Options are the following: share (or warrant / option for shares), items, tokens, rewards or cryptocurrency.

Tokens can have a different purpose. They can represent the rights of use of the product, or property rights tied to some internal value.

4. Transparency of management. It is necessary to think over various aspects of management, regardless of whether stakeholders are involved actively (e.g. voting, management, rule making, rule checking, decision making, reporting, and regulation), or passively (e.g. sense of responsibility, evaluation, respect, fair compensation (10). All this is designed to ensure transparency in management.

5. Profit. In the ordinary corporation, profit distribution or participation in dividends takes place. In DAO, benefits to participants may include voting or special rights, or granting of special status. Ultimately, the growth of value should happen due to an internal high valuation of capital, either in the form of a cryptocurrency or cryptographically protected tokenization.

6. Crypto-technology. Protocols and platforms based on the blockchain and cryptocurrency are a means of achieving a consensus mechanism. The blockchain and cryptographic evidences ensure the irrefutability, verifiability and truthfulness of all transactions and smart programs based on the decentralized open source consensus and decentralized protocols of trust. Ethereum, Bitcoin, general purpose protocols, and special purpose protocols (for example, Dash) are the examples of such protocols.

There are three additional functional components that should be included in the technology platform:

a) the level of user data, assuming that the data belong to the user and are only available in a specific aggregate or hidden form of the DAO;

b) smart programs that are real mechanisms of transactions;

c) various application programming interfaces (APIs) for interacting with value-added services or partners that are auxiliary to the DAO.

The key goal of DAO is to create value or products, and this requires a certain connection between the user's actions and the resulting consequences of these actions for the total value of the organization that is symbolized by the value of the cryptocurrency, which underlies it. This is exactly what entrepreneurial creativity should be, aimed at developing business models.

DAO should be tied to the cost. The sale of the cryptocurrency allows the DAO beginning its movement. In the end, the new DAO is like a startup. It requires the adaptation of the product to the market, the implementation of the business model and a large number of users (customers) to move forward. At the early stage, many assumptions are made, and the DAO 
may resemble a science-fiction project, until the product or service proves to be viable on the market. Market resilience and not the success of initial fundraising is the evidence of success.

The creation of DAO is a step-by-step and gradual process of constructing a building that cannot be artificially accelerated.

Companies may not fully comply with the idea of DAO. Companies can only partially implement the DAO principles. There is no right or wrong way of implementing the blockchain inside large organizations. There are different approaches. The launch starts with a clean sheet of paper and without any baggage. Therefore, it is not easy to make an organization a conscious, well-informed supporter of the blockchain and a connoisseur of its capabilities. As a rule, this starts to happen after the interested parties appear in the company, and after the external pressure of the market is added to the interest of progressive employees.

One can draw an analogy between the introduction of reengineering in the early 1990s, and the introduction of the blockchain. Reengineering of business was a common approach in the 1990s. The difference between the epoch of reengineering and the early period of the blockchain is that the approaches of reengineering have mostly been written, and most of the work was devoted to the implementation.

In the early 1990s, the information technology was the main revolutionary technology. Technologies of the corporate blockchain grew and matured until 2016. Now it is time to introduce the blockchain in companies, for which it is useful to borrow the idea of "reengineering king", which was used in the 1990s. "The king of the blockchain" should understand business operations and the role of reengineering of the business process, as well as being the blockchain enthusiast. He should be responsible for removing obstacles in the organization, for promoting education, supervising projects and sharing best practices, and supervising the implementation of the blockchain in the organization. This is a difficult task, because it consists in finding and destroying old processes instead of automating or optimizing what is being done at the moment.

W. Mougayar is devising 13 strategies for corporate executives aimed at solving various problems [5]. Let us name these strategies:

1. The strategy of combating anchors.

The problem is that the blockchain redefines the past. Large companies always struggle with their obsolete applications, because they can be anchors that interfere with the advent of new technologies.

2. Creating a strategic advantage based on the blockchain.

The blockchain is the strategic platform of IT. The blockchain in its fullest form is the new main platform for software development. Therefore, it is of an increasingly strategic nature. A strategic approach means that it does not only reduce costs and increase the speed of transactions. This means that it needs to find strategic use that can provide for a competitive advantage.

3. Strategy of creating competencies.

It is used for the full deployment of solutions on the blockchain inside of the company, such as education, discovery, design, development and management.

Education is aimed at studying the basic functionality of the blockchain and what it allows in general.

Discovery answers the question: where can the blockchain be applied and what can we do with it?

Design decides what functional solutions we will need to use the potential that we saw at the initial stage? How will this affect influence what we do, including the business process, contractual and legal requirements?

Development is aimed at creating software, integrating and implementing technology.

Management includes ongoing software maintenance, support, iterative evolution, new features and updates. 
Most companies cannot develop expertise in all these areas, but they can cooperate with external firms on specific aspects of these steps. Knowing how to program the blockchains will be a necessary competence, no less important than programming the Internet applications.

4. The strategy of choosing partners.

Each organization has its own starting point, based on its resources and capabilities, so the chosen approach will depend on the specific situation.

5. The strategy of internal integration.

The problem is that when the blockchain is introduced into an existing system, it must be integrated with the existing corporate systems. However, the blockchain also has the potential to replace some internal processes. It is easier to start using the blockchain in some new segments without internal integration.

6. The strategy of developing the blockchain as a platform for joint services.

It is possible to create joint services of the blockchains either at the vertical level (for example, in a specific financial services application), or at the horizontal level (for example, a common record verification service).

7. The strategy of improvement.

For startups, it is clear that the blockchain is a destroyer, but for large companies, the first likely scenarios are to deploy the blockchain technology to improve existing operations.

8. The strategy of the blockchain as a new database.

The blockchain as a new database.

9. The strategy of the blockchain platform.

The blockchain is considered as a service.

10. The strategy of training.

A proactive approach to training employees of various departments to the blockchain technology is introduced.

11. Strategy of the proof.

Large companies often use the "proof of concepts" (POCs) approach. The sense is to implement end-to-end projects of a smaller scale, in which one can see the results of using the blockchain with real users.

12. Strategy of reengineering.

If the blockchain technology is not yet ready, one can start reengineering business processes while the technology is being prepared.

13. Strategy of cases.

It means to be engaged not only in improving with the help of the blockchain, but in thinking in the context of innovation.

Thus, the blockchain is a new paradigm for business. To develop the blockchain strategy, it is necessary to keep three elements in mind simultaneously: solving problems, identifying opportunities and implementing them. Such is the triad of sanity for the organizational strategy of the blockchain.

Another triad of action in moving towards decentralized organizations is to provide flexibility, openness and consensus. It is necessary to determine the tasks, distribute the workload among people who are able and ready to fulfill them, come to an agreement on their roles, duties and remuneration, and then fix them in the form of explicit and detailed self-fulfilling agreements called smart contracts $[10,11]$.

Let us highlight the opportunities for entrepreneurship development, which gives the use of DAO and smart contracts:

- Distributed autonomous organizations will become viable, with independent operations and creating value for users who are directly tied to services and financial rewards.

- Spontaneous and reliable trade will occur between equal partners, without central intermediaries, and with minimal or no difficulty. 
- Distribution of content and competencies will be irrefutably signed on the blockchain.

- Anyone can implement the business logic and contract with other people, and easily apply them to the blockchain.

- The popularity of services will increase, in which users receive cryptocurrency by performing routine services.

Further research on the topic of DAO should be connected with identification of the costs of introducing DAO and determining the effectiveness of DAO functioning in comparison with centralized organizations.

\section{References}

1. The program "Digital Economy of the Russian Federation" (Approved by the Government Regulation No. 1632-r (http://static.government.ru/media/files/9gFM4FHj 4PsB79I5v7yLVuPgu4bvR7M0.pdf, 2017)

2. The promise of the blockchain. The trust machine. The technology behind bitcoin could transform as the economy works (https://www.economist.com/news/leaders/21677198technology-behind-bitcoin-could-transform-how-economy-works-trust-machine, 2015)

3. M. Y. Veselovsky, M. A. Izmailova, A. V. Bogoviz, S. V. Lobova, Y. V. Ragulina, Quality - Access to Success, 19(163), 30-36 (2018)

4. N. S. Mrochkovsky, ITMO: Econ. and Eco-management, 2, 105-112 (2018)

5. U. Mogaiar, Blocking for business (Eksmo, Moscow, 2018)

6. What are decentralized autonomous organizations and why they are needed (https://forklog.com/chto-takoe-detsentralizovannye-avtonomnye-organizatsii-izachem-oni-nuzhny, 2018)

7. The main leakage of 2017 (https://www.infowatch.ru/analytics/digest/19546, 2018)

8. The data flows off by a megapress (https://www.rbc.ru/newspaper/2017/03/24/58d2bbb 39a794721422e1588, 2018)

9. Results of the competition for the best DAO (https://forklog.com/dao-contest-results, 2018)

10. D. Tapscott, A. Thapscott, Technology of blockade: what drives the financial revolution today (Eksmo, Moscow, 2017)

11. M. Y. Veselovsky, M. A. Izmailova, A. V. Bogoviz, S. V. Lobova, A. N. Alekseev, Quality - Access to Success, 19(162), 60-66 (2018) 